\title{
ENERGÍA CINÉTICA PRODUCIDA EN FOTOELECTRONES DE CU INDUCIDO POR FOTONES DE ALTA ENERGÍA DE LA LUZ
}

\section{KINETIC ENERGY PRODUCED IN CU PHOTOELECTRONS INDUCED BY HIGH ENERGY PHOTONS OF LIGHT}

\author{
Grave-Capistrán, M. A. * García León R. A.1 Quintero-Quintero. W**. \\ *Instituto Politécnico Nacional, SEPI-ESIME-Zacatenco. Col. Lindavista C.P. \\ 07738, México ${ }^{*, * *}$ \\ **Facultad de Ciencias Admistrativas y Economicas. \\ Universidad Francisco de Paula Santander, \\ Colombia \\ mgravec1200@alumno.ipn.mx.
}

\begin{abstract}
Resumen: El efecto fotoeléctrico en el cobre ha sido estudiado desde distintos ámbitos alterando cualquiera de sus propiedades. Para este estudio en particular el cobre se considera en su estado natural y contemplando la función de trabajo del metal se propusieron 5 escenarios de estudio en los que se varía la frecuencia de onda de la luz para analizar y cuantificar la energía cinética en los fotoelectrones emitidos. Analíticamente se obtuvieron los valores para cada caso de estudio y numéricamente se generaron gráficas en las que se observa el comportamiento de la energía cinética frente a la frecuencia y longitud de onda inducidas. El espectro electromagnético UV es el último en el que se puede presentar el efecto fotoeléctrico en el $\mathrm{Cu}$, el valor obtenido es de $4.562 \llbracket \mathrm{X} 10 \rrbracket \wedge(-17) \mathrm{J}$ y representa la menor energía cinética generada en los fotoelectrones mientras que $6.625 \llbracket$ X10 $\rrbracket \wedge(-10) J$ es el valor máximo en radiación gamma $(\gamma)$..
\end{abstract}

Palabras clave: Cobre, Energía Cinética, Fotoeléctrico, Fotoelectrón, Frecuencia.

\begin{abstract}
Photoelectric effect on copper has been studied from different fields modifying its properties. For this specific study, copper is considered in its natural state and its work function; 5 study scenarios were proposed which ones the wavelength of light is changing in order to analyse and quantify the kinetic energy in the emitted photoelectrons. Analytical values were obtained for each case study and numerical graphs were generated in which the behaviour of the kinetic energy is observed against the frequency and wavelength induced. The UV electromagnetic spectrum is the last case in which the photoelectric effect can be presented on copper $(\mathrm{Cu})$ since the threshold frequency and work function of the metal allow it, the value obtained is and represents the lowest kinetic energy generated in the photoelectrons while is the maximum
\end{abstract} value in gamma radiation.

Keywords Copper, Frequency, Kinetic Energy, Photoelectric, Photoelectron 


\section{INTRODUCCIÓN}

El cobre es uno de los metales más utilizados en el mundo en aplicaciones industriales y de la vida cotidiana, la respuesta de interacción con otros agentes lo hacen un material para el estudio de sus propiedades mecánicas, físicas y químicas ante una perturbación espontanea.

El efecto fotoeléctrico ha sido observado desde el punto vista cuántico para comprender el fenómeno. A partir de la constante de Planck, la frecuencia de la luz y la función del trabajo de los metales es posible determinar cuantitativamente la energía cinética de los fotoelectrones expulsados de la superficie del mismo, si la energía del fotón es menor al de la función del trabajo del cobre ningún electrón será emanado ya que no se absorbe suficiente energía para liberarse del metal y lograr generar energía cinética. Se ha establecido que el potencial de frenado del metal es proporcional a la frecuencia de la luz incidida. La aplicación de rayos Roentgen en la superficie de un compuesto químico que involucra al cobre, empleando la técnica de espectroscopía fotoelectrónica de rayos " $\mathrm{X}$ " de presión ambiental se demostró después de la reacción química en cada una de las superficies empleadas la energía cinética presenta un crecimiento en el electrón en relación a la frecuencia de la luz inducida. Por otro lado, al bombardear la superficie del cobre con radiación $\gamma$ se tiene un aumento en los fotoelectrones expulsados del orden de los Kiloelectrovoltios por lo que el nivel de radiación en el material es considerable y proporcional en el aumento de la frecuencia incidida. [1 - 4]; (L Tangarife et al., 2017)

Otros estudios reportan que el comportamiento de la energía cinética en los fotoelectrones también se ve afectada por el cambio de temperatura en la superficie de los metales, en particular un estudio efectuado en un compuesto por cobre y grafeno demostró que, aunque los niveles de temperatura fueran bajos o muy altos el equilibrio entre energía potencial y cinética no se lograba. Las deformaciones plásticas sobre la superficie del cobre afectan a los fotoelectrones expulsados al incidir luz y a su vez a la energía cinética y potencial de todos los átomos. La energía cinética aumenta en relación a la velocidad con la que se deforma plásticamente al material dependiendo de la estructura cristalina del mismo. Un análisis en las nano partículas de cobre ante una alteración por energía solar térmica demostró que las nano partículas adquieren mayor energía cinética para moverse libremente en la capa de la superficie del
$\mathrm{Cu}$ y obtuvieron incrementa la frecuencia de colisión en las nano partículas alterando las propiedades fisicoquímicas del material. Una simulación dinámica molecular en las nano partículas de cobre y platino por variación de temperatura comprobó que cada una de las partículas fluctúa en la energía cinética que se genera. Además, concluyen que con el aumento de la fuerza y la velocidad la temperatura incrementa proporcional y positivamente con la energía cinética en la superficie del material [5 - 8], (J Plaza, M Núñez, 2017); (O Suarez at el, 2018)

El estudio de efectos mecánicos en el espacio y tiempo con cualidades ontológicas permite establecer las leyes y relaciones matemáticas que gobiernan.

La mecánica clásica y mecánica newtoniana han permitido profundizar el análisis y estudio de la relatividad en fenómenos físicos como es el caso del efecto fotoeléctrico [9 - 10].

Mediante simulaciones numéricas y cálculos analíticos se ha cuantificado la energía cinética en los fotoelectrones emanados desde la superficie del cobre compuesto o en diferentes arreglos cristalinos dependiendo de la frecuencia de la onda. Experimentalmente se han alterado las propiedades mecánicas del metal y se ha observado el comportamiento de los átomos con apoyo de instrumentos ópticos y se concluido que a través de este metal es posible generar corriente a partir de la energía cinética generada.

Aunque se ha estudiado el comportamiento de le energía cinética en el cobre en diferentes escenarios y acompañado de distintas composiciones no se ha obtenido una relación descendente de la energía cinética en función de la frecuencia de la onda. La energía del fotón emitido es factor importante para concebir el efecto fotoeléctrico y no se ha establecido el punto en el que hay una menor cantidad de energía cinética en los fotoelectrones emitidos desde la superficie del metal.

\section{MATERIALES Y METODOS}

El efecto fotoeléctrico se produce cuando a la superficie de un metal se incide radiación electromagnética que depende de la frecuencia de la onda de la luz para emitir electrones con modificación en sus propiedades entre ellas, la de la energía cinética. Al paso del tiempo se ha tenido cada vez más aplicación de este efecto ya que se han estado empleando compuestos que alteran la estructura molecular de los metales base.

El propósito de esta investigación radica en el análisis de la energía cinética durante el proceso 
del efecto fotoeléctrico en el cobre en estado sólido a través de cálculo analítico y solución gráfica, para tal caso el trabajo se divide la siguiente forma: Se considerará al cobre en su estado natural; se tomarán en cuenta 5 valores de frecuencia de onda en orden ascendente hasta detectar el valor mínimo en el que se genera el efecto; empleando MatLab® versión estudiantil se establecerán las ecuaciones de gobierno y se obtendrán de forma gráfica los valores y el comportamiento de las variantes de la energía cinética, la Tabla 1 muestra los parámetros de la luz que se emplearan desde radiación $\gamma$ hasta rayos UV considerando como características principales la frecuencia de onda y la longitud de onda.

\begin{tabular}{c|c|c|c}
\multicolumn{3}{c}{ Tabla 1. Parámetros De Evaluación De La Luz En } \\
$\begin{array}{c}\text { Escenari } \\
\text { O }\end{array}$ & $\begin{array}{c}\text { Espectro } \\
\text { electromagnético }\end{array}$ & $\begin{array}{c}\text { Frecuencia } \\
\text { de onda } \\
{[\mathrm{Hz}]}\end{array}$ & $\begin{array}{c}\text { Longitud } \\
\text { de onda } \\
{[\mathrm{m}]}\end{array}$ \\
\hline I & Radiación Gamma & $10^{24}$ & $10^{-16}$ \\
\hline II & Radiación Gamma & $10^{22}$ & $10^{-14}$ \\
\hline III & Radiación Gamma & $10^{20}$ & $10^{-12}$ \\
\hline IV & Rayos Roentgen & $6.0 \times 10^{18}$ & $10^{-10}$ \\
\hline V & UV & $7.0 \times 10^{16}$ & $10^{-8}$
\end{tabular}

Partiendo de la propuesta de Einstein, en la que la luz se comporta como una corriente de partículas denominadas fotones y donde la energía se plantea en la ecuación 1:

$\mathrm{E}=\mathrm{h} \cdot \mathrm{v}$

Donde

E: energía del fotón en Joules (J)

h: constante de Planck $=6.626$ X10-34 J.s

v: frecuencia de la luz en Hertz $(\mathrm{Hz})$

Ahora se considera la función de trabajo del metal $(\Phi)$ de estudio, en el cobre el valor es de:

$$
\Phi \_\mathrm{Cu}=7.53 \mathrm{X} \text { 【10】^(-19) J }
$$

Finalmente, para obtener la energía cinética en los fotoelectrones emitidos por el efecto fotoeléctrico se plantea la siguiente ecuación.

KEelectrón $=h \cdot v-\Phi$
Substituyendo los valores de la constante de Planck y la función del trabajo del cobre en la ecuación 2 queda como variable la frecuencia de onda de la luz. Calculando los resultados para cada uno de los casos se obtendrá una relación entre la frecuencia empleada y la cantidad de energía cinética que se produce; (A Niño, 2018)

\section{RESULTADOS Y DISCUSIONES}

Con base en la ecuación (2) y el parámetro del metal se evalúan cada uno de los escenarios de forma analítica para obtener el valor de la energía cinética de los fotoelectrones expulsados desde la superficie del metal, la Tabla 2 presenta los resultados obtenidos.

\section{TABLA 2. Resultados De La Energía Cinética En Los Fotoelectrones Emitidos Del Cu}

\begin{tabular}{c|c|c}
$\begin{array}{c}\text { Escenari } \\
\text { O }\end{array}$ & $\begin{array}{c}\text { Espectro } \\
\text { electromagnético }\end{array}$ & $\begin{array}{c}\text { Energía } \\
\text { cinética del } \\
\text { fotoelectrón [J] }\end{array}$ \\
\hline I & Radiación Gamma & $6.625 \times 10^{-10}$ \\
\hline II & Radiación Gamma & $6.625 \times 10^{-12}$ \\
\hline III & Radiación Gamma & $6.625 \times 10^{-14}$ \\
\hline IV & Rayos Roentgen & $3.974 \times 10^{-15}$ \\
\hline V & UV & $4.562 \times 10^{-17}$
\end{tabular}

Los resultados de la Tabla 2 vislumbran la obtención de un gráfico lineal creciente entre la relación de la energía cinética y la frecuencia de onda empleada a través de los 5 escenarios, esto debido a que la disminución proporcional de la energía depende de la frecuencia de onda de los rayos inducidos.

Los resultados obtenidos de cada una de las ecuaciones en el programa de computo coinciden completamente con los del método analítico, de esta forma se valida la información que corresponde a cada uno de los escenarios para el $\mathrm{Cu}$.

En la Figura 1 se observa el comportamiento lineal considerando los parámetros y resultados de cada uno de los escenarios planteados 


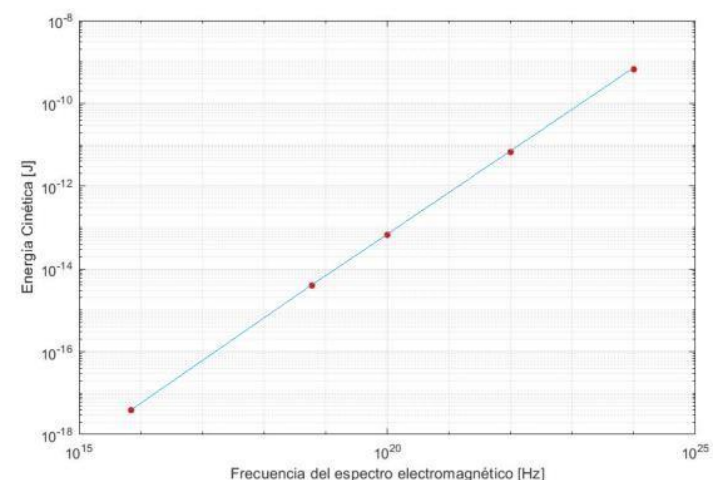

Fig. 1.- Respuesta de la energía cinética en función de la frecuencia en los escenarios I a V

Los resultados del escenario $\mathrm{V}$ representan el valor mas próximo en el que los fotoelectrones pueden llegar a ser emitidos desde la superficie del metal esto debido a que el valor de la energía del fotón no supera el valor de la función del trabajo del cobre y para que el efecto fotoeléctrico suceda se debe cumplir esta condición.

A partir de la fecuencia de la luz incidida de este escenario no es posible la emisión de fotoelectrones y como consecuancia no existe energía cinética cuantificable. Como valor máximo de energía cinética se obtuvó registrado $\mathrm{J}$ que corresponde a radiación gamma incidida y como valor mínimo es incidiendo rayos

UV cuyo valor es de $\mathrm{J}$.

Por otro lado, la relación entre la energía cinética generada y la longitud de onda de de la luz presentan un comportamiento inversamente proporcional ya que el valor de la longitud de onda es muy pequeño, la emisión de fotoelectrones es mayor cuando se encuentran entre el escenario I al III por lo tanto la energía cinética comparte el mismo comportamiento pero la longitud de onda en radiación es corta.

El escenario IV y V comparten las caracteristcas de radiación pero la diferencia entre la longitud de onda y frecuencia de la luz es significativa y esto afecta de forma notable la emisión de fotoelectrones en la superficie del $\mathrm{Cu}$.

La Figura 2 muestra la relación entre la longitud de onda de radiación, Roentgen y UV y la energía cinética generada por cada uno de los parámetros ya mencionados, el comportamiento es debido a que la longitud de onda es corta dentro de los escenarios planteados pero la energía cinética

presenta una cantidad considerable en los fotoelectrones emitidos.

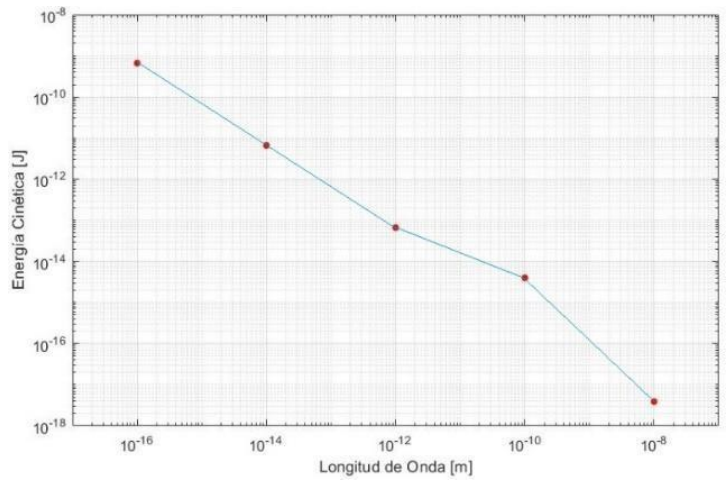

Fig. 2.- Respuesta de la energía cinética en función de la longitud de onda en los escenarios I a V

A partir del espectro electromagnético de luz visible no es posible obtener un valor cuantificable de la emisión de fotoelectrones y de energía cinética en los mismos debido a que el efecto fotelectrico no se genera. Aunque la frecuencia de la luz es notable en la luz visible no tiene efectos en el cobre ya que dependen del parámetro de la función del trabajo.

Entre el escenario I y IV que involucra radiación $\gamma$ y Roentgen se presenta menor longitud de onda frente a la energía cinética generada. Con esta información se comprueba el comportamiento del efecto fotoeléctrico en metales en donde la energía del fotón no supera el valor de la función del trabajo.

La literatura abierta comprueba que el comportamiento de la energía cinética durante el efecto fotoeléctrico en cualquiera de los metales sin alterar es lineal. Cuando la frecuencia de onda inducida en la superficie el del $\mathrm{Cu}$ es alta se obtiene mayor energía cinética en los fotoelectrones expulsados.

Meier-Jaramillo, E. desarrolló la aplicación del efecto fotoeléctrico en diferentes metales, los resultados que obtuvo en cada metal coinciden gráficamente entre la energía cinética y la frecuencia de forma lineal. Aunque el autor se enfocó en un valor de la frecuencia de la onda inducida al cobre los resultados comparten similitud con los del presente trabajo de investigación.

Otro estudio elaborado por Corasa, I. en el que efectúa una comparación entre placas de plomo y

cobre bombardeadas con rayos gamma consiguió establecer una relación entre la energía cinética producida en la que el comportamiento de la 
energía cinética es lineal proporcional a el nivel de la frecuencia del espectro electromagnético inducido.

El trabajo de Escudero, J. involucra la ecuación para el cálculo de la energía cinética en los fotoelectrones, la constante de Planck es utilizada y aunque la frecuencia la calculó a partir del cociente de la velocidad de la luz entre la longitud de onda los resultados en la energía cinética de los fotoelectrones son similares a los del trabajo desarrollado.

Cada investigación encontrada en la literatura abierta tiene sus propósitos y objetos de estudio de interés por cada autor, en este trabajo se estudiaron 5 escenarios en los que la frecuencia de la onda se considera desde la radiación gamma hasta los rayos UV ya que es en los que cuales es posible generar el efecto fotoeléctrico y los resultados son particularmente estrechados con los de otros autores fundamentando los resultados tanto analíticos como gráficos de este trabajo

\section{CONCLUSIONES}

Durante la elaboración de este trabajado ha quedado establecido y cuantificado el valor de la energía cinética a través del espectro electromagnético de forma analítica y en solución gráfica. Con programas especializados se comprueba en las gráficas que la emisión de fotoelectrones desde la superficie del cobre es posible hasta el bombardeo con rayos UV ya que la energía del fotón es 4.638 [ X10 \^(-17) y a su vez es mayor al valor de función de trabajo del cobre $(7.53 \mathrm{X}$ [10】^(-19)) permite que fotoelectrones desde la superficie puedan ser expulsados con energía cinética. El movimiento de los fotoelectrones expulsados en el medio durante el proceso fotoeléctrico permitirá efectuar un análisis desde el estado de reposo de los mismos hasta cuando su velocidad afecte la energía cinética generada

La función de trabajo del cobre permite la emisión de fotoelectrones desde la radiación gamma hasta los rayos UV estos contienen energía cinética cuantificable para estudios sobre la mecánica clásica y de la mecánica cuántica, en otro panorama donde se involucra a la temperatura es

posible observar un comportamiento diferente en el $\mathrm{Cu}$. La frecuencia de onda de la luz a partir de la visible hasta las ondas de radio largas es mayor al de función de trabajo del $\mathrm{Cu}$ por lo que la emisión de fotoelectrones es imposible. Incidir mayor frecuencia en la superficie del metal genera mayor cantidad de energía cinética en los fotoelectrones, pero la longitud de onda es corta debido a esto se obtiene una cantidad importante de transmisión de corriente eléctrica en el medio, en su mayoría se ocupa para alimentar baterías.

Es importante conocer el comportamiento nanoestrucural de los metales afectados por agentes externos, en este caso el cobre cuenta con un gran número de aplicaciones y se debe considerar como es la alteración de sus propiedades ante perturbaciones y que lleguen a afectar su desempeño.

\section{RECONOCIMIENTO}

El autor agradece al Instituto Politécnico Nacional y al Consejo Nacional de Ciencia y Tecnología (CONACyT) por el apoyo brindado para la concepción del presente trabajo de investigación.

\section{REFERENCIAS}

AB Niño (2018) Micro turbina Peltón, una solución real de energía para zonas no interconectadas (ZNI). Revista Colombiana de Tecnologías de Avanzada, ISSN: 16927257.

Baran, E., Leonid, L., Cheng Hao Wu., Hendrik, B., Gabor A. \& Miquel, S.. (2015). Reaction of CO with Preadsorbed Oxygen on LowIndex Copper Surfaces: An Ambient Pressure X-ray Photoelectron Spectroscopy and Scanning Tunneling Microscopy Study. The Journal of Physical Chemistry, 1, pp. B-E.

JEG Plaza, MAR Nuñez, (2017) Formación en competencias específicas para la industria del software colombiano. Experiencias del uso del aprendizaje basado en proyectos. Revista Colombiana de Tecnologías de Avanzada, ISSN: 1692-7257

Coarasa-Casas I.. (2015). Atenuación de la radiación gamma con blindajes de plomo y cobre.

Meier-Jaramillo, E.. (2016). Influencia de la función de trabajo en el efecto fotoeléctrico. ResearchGate, 1, pp. 3, 7-10. 
Escudero, C., Jaime, E. \& González, S. (2016). Hacia la conciencia cuántica a partir del efecto fotoeléctrico. Enseñanza de las Ciencias, 1, pp. 188-191.

L Tangarife, M Sánchez, M Rojas (2017). Modelo de interventoría de tecnologías de información en el área de conocimiento de la gestión del alcance de PMBOK® y alineado con ISO 21500 y COBIT®. Revista Colombiana de Tecnologías de Avanzada, ISSN: 1692-7257

Yang, H., Lei L., Xiao-Cheng Z. \& Jingchao Z.. (2015). Tuning thermal contact conductance at graphene-copper interface via surface nanoengineering. Royal Society of Chemistry, 1, pp. 7, 11 .

O Suarez, C Vega, E Sánchez, A Pardo. (2018) Degradación anormal de p53 e inducción de apoptosis en la red P53-mdm2 usando la estrategia de control tipo pin. Revista Colombiana de Tecnologías de Avanzada, ISSN: 1692-7257.

Jia, L., Bin, Liu., Hao, L., Qihong, F., Youwen, L. \& Yon, L.. (2016). A molecular dynamics investigation into plastic deformation mechanism of nanocrystalline copper for different nanoscratching rates. ELSEVIER, 1, pp. 73-75.

Saw, C. L. \& Hussain, H.. (2016). Evaluation of copper nanoparticles - Paraffin wax compositions for solar thermal energy storage. ELSEVIER, 1, pp. 274-275.

Davood, T., Majid, M. \& Masoud, A.. (2016). Molecular dynamicsimulationofCopperandPlatinumnano particles Poiseuille flow inananochannels. ELSEVIER, 1, pp. 3-6.

Raúl Molina Gil. (2015). De lo subatómico a lo inmenso: sobre la influencia de la teoría de la relatividad y la mecánica cuántica en lo fantástico. Brumal, 3.

Lecea-Blanco R.. (2016). Relatividad y relativismo en la ciencia. pp.64-66.Ogata, K. (2004). Ingeniería de Control Moderna, Prentice Hall, Cuarta edición, Madrid.

Clymer, J. R. (1992). "Discrete Event Fuzzy 